\title{
Publisher's Note: Modeling the excitation of graphene plasmons in periodic grids of graphene ribbons: An analytical approach [Phys. Rev. B 94, 195421 (2016)]
}

\author{
P. A. D. Gonçalves, E. J. C. Dias, Yu. V. Bludov, and N. M. R. Peres
}

(Received 20 December 2016; published 30 December 2016)

DOI: 10.1103/PhysRevB.94.239908

This paper was published online on 14 November 2016 with an omission of an author affiliation. N. M. R. Peres's additional affiliation should read as “4 QuantaLab, University of Minho, PT-4710-057, Braga, Portugal.” The affiliations have been corrected as of 20 December 2016. The affiliations are incorrect in the printed version of the journal. 